
\title{
$\begin{array}{ll}\text { Research Square } & \text { Preprints are preliminary reports that have not undergone peer review. } \\ \text { They should not be considered conclusive, used to inform clinical practice, } \\ \text { or referenced by the media as validated information. }\end{array}$ \\ Feasibility and reproducibility of the fully-automated strain analysis in a healthy population: a comparative study with the manual analysis
}

Yingying Liu ( $\sim$ maggielyy21064@163.com )

Shenzhen People's Hospital https://orcid.org/0000-0002-6792-8933

\section{Guijuan Peng}

Shenzhen People's Hospital

\section{Shuyu Luo}

Shenzhen People's Hospital

Xiaofang Zhong

Shenzhen People's Hospital

Xiaoxuan Lin

Jinan University First Clinical Hospital: Shenzhen People's Hospital

Zheng Yingqi

Shenzhen People's Hospital

Jinfeng Xu

Shenzhen People's Hospital

Lixin Chen

Shenzhen People's Hospital

\section{Research Article}

Keywords: strain, speckle tracking echocardiography, automatic assessment, left ventricle, right ventricle

Posted Date: February 1st, 2022

DOI: https://doi.org/10.21203/rs.3.rs-1158594/v1

License: (c) (1) This work is licensed under a Creative Commons Attribution 4.0 International License.

Read Full License 


\section{Abstract}

Purpose: The feasibility of strain analysis in clinical practice was hindered by two reasons: timeconsuming and a lack of specific analytic packages for the right ventricle and atria. A novel software called Autostrain automatically tracks the myocardium for strain analysis. This study aimed to evaluate the feasibility and reproducibility of the automatic analysis method in a healthy population and compare it with the manual method.

Methods: Two trained doctors performed strain analysis of the left ventricle (LV), left atrium (LA), right ventricle (RV), and right atrium (RA) using automatic and manual methods (Qlab software) in 159 healthy volunteers. The reproducibility of automatic analysis between two consecutive cardiac cycles was assessed.

Results: The feasibility of automatic analysis in LV, LA, RV, and RA were 145/152(95.4\%), 151/152(99.3\%), 143/152(94.1\%), 149/152(98.0\%), respectively. For the measurement of global longitudinal strain (GLS), a good correlation was shown between the automatic results of two consecutive cardiac cycles $(r=0.742-0.854, P \bigotimes 0.001)$. No significant differences in LV-GLS between the two methods were shown $(-21.1 \% \pm 2.4 \%$ vs. $-20.8 \% \pm 2.4 \%, P=0.05)$. The RV free-wall, LA, and RA global longitudinal strain measured by the automatic software significantly differed from the manual analysis. The automated method had a significantly shorter analysis time than the manual method.

Conclusion: The novel, fully-automatic myocardium strain analysis software can provide a rapid assessment with high reproducibility, which is more feasible and adaptable in clinical practice. It should be noted that strain values measured by automatic and manual analysis cannot be used interchangeably.

\section{Introduction}

In the past decade, two-dimensional speckle tracking imaging (2D-STI) has been widely studied and applied in the assessment of myocardial deformation, especially in longitudinal strain. Longitudinal strain is a sensitive parameter for evaluating the myocardial function, which is superior to the conventional echocardiographic parameters (such as left ventricular ejection fraction, left atrial volume index). It is also of greater value than the conventional parameters in risk stratification, clinical decisionmaking, and prognosis analysis [1-4].

Although several studies have demonstrated the feasibility of 2D-STE in assessing longitudinal myocardial deformation, its feasibility in clinical practice was hindered by several reasons. Firstly, most speckle tracking analyses were developed based on the left ventricle (LV) model. There was still a lack of specific packages for analyzing the right ventricle (RV) or atria. It is controversial whether the LV-based software package would be reliable when used on RV and atria[5]. Secondly, most of the current strain analysis software is limited to off-line analysis and complicated to operate, which is inconvenient and time-consuming[6]. The fully-automatic strain analysis software contains specific packages for RV and left atrium (LA), and its online analysis function may help promote the technology in clinics. 
The study aimed to evaluate the feasibility and reproducibility of the fully automated analysis software in a healthy population and compare it with the usual manual strain analysis.

\section{Materials And Methods 2.1 Patient Population}

Patients who underwent regular physical examination in our hospital from March 2021 to April 2021 were selected. Those with a history of atrial fibrillation or flutter, coronary heart disease, congenital heart disease, moderate or severe valvular disease, heart failure, hypertension, or kidney failure were excluded. All participants underwent electrocardiogram (ECG) examination, and those with ECG abnormalities were excluded. Informed consent was obtained from each patient; the study protocol conforms to the ethical guidelines of the 1975 Declaration of Helsinki as reflected in a priori approval by the Ethics Committee of Shenzhen People's Hospital.

\subsection{Image acquisition and strain analysis}

Patients were placed in the left decubitus position and monitored by an electrocardiogram. Videos of three standard sections (apical four-chamber, two-chamber, and three-chamber views) were recorded using both Philip Epic7C and Philip CVx ultrasound system (Philips Healthcare, Bothell, Washington, USA) with a $1-5 \mathrm{MHz}$ transducer (S5-1). At least four cardiac cycles were recorded in each video. All the videos were saved in Digital Imaging and Communications in Medicine (DICOM) format. The left ventricular ejection fraction (LVEF) was measured using the biplane Simpson's method.

According to the European Society of Cardiography [7], LV global longitudinal strain (GLS) was measured using the apical two-chamber, three-chamber, and four-chamber views. RV strain was measured on the apical four-chamber view or the RV-focused view. LA and right atrium (RA) strain were measured on the apical four-chamber view. All cases were analyzed using the AutoStrain software (Philips Healthcare) and the Qlab software (Qlab 9.1, Philips Healthcare). All the analyses were completed by two trained doctors independently. The analysts were blinded to the measurements performed by each other and to the clinical data.

After selecting appropriate sections for analysis, the AutoStrain software can automatically recognize, segment, and track LV, RV, and LA myocardium, and the strain curves were simultaneously calculated (See Fig. 1-2). The LA-specific analytic package (LA Strain) was used to obtain the RA strain. After manually determining the right atrial apex and tricuspid annulus, the software automatically tracked the intima throughout the cardiac cycle (see Fig. 2). Two consecutive cardiac cycles were randomly selected from the video for analysis, and the same cardiac cycles were used in the manual analyses for comparison.

The commercially available Qlab CMQ software was used for manual analysis. The LV-specific strain analysis software was used for analyzing all chambers. After manually selecting the frames (the end- 
systolic and the end-diastolic frame) and setting the reference points (the position of the apex, the anterior, posterior, medial, and lateral border of the annulus), the software divided the region of interest into six segments and tracked the myocardial motion frame by frame (See Fig. 1-2).

For both methods, all tracking was reviewed to ensure that it was a proper presentation of atrial and ventricular motion. Segments with poor tracking would be readjusted until a better tracking quality was achieved (See Supplementary Figure 1-2). If satisfactory tracking was not attainable in more than two segments in one video, the video was regarded as "failed"[8]. The measurement time was recorded from the initial selection of images for analysis to the proper presentation of the global longitudinal strain curve for each case in 50 randomly selected cases.

\subsection{Interobserver and interobserver variability}

A subset of 20 randomly selected cases were used for intra-observer and inter-observer variability assessment. The cases were measured by two trained observers using both methods independently and measured twice during a 30-day interval.

\subsection{Statistical analysis}

Continuous variables are expressed as mean \pm standard deviation or median (interquartile range), and categorical variables are shown as percentages. The paired t-test was used to compare the strain values measured by two different methods. Linear correlation analysis and Bland-Altman analysis were used to assess the consistency of the results between the two methods. Linear correlation analysis was also used to compare the strain values between two consecutive cardiac cycles. SPSS version 18.0 was used for statistical analysis. The inter-observer and intra-observer variability were calculated with the interclass correlation coefficient (ICC). A p-value less than 0.05 was considered statistically significant.

\section{Results}

\subsection{Study subjects}

A total of 159 cases were collected in this study, among which 7 cases were excluded due to bad image quality, and 152 cases were eventually included. The patient characteristics are shown in Table 1. For automatic strain analysis, the success rates for LV, LA, RV, and RA were 145/152 (95.4\%), 151/152(99.3\%), 143/152 (94.1\%), and 149/152 (98.0\%), respectively, and the GLSs were $-21.0 \% \pm 2.5 \%$, $48.0 \% \pm 10.0 \%$ (the reservoir strain), $-26.3 \pm 4.9 \%$, and $41.1 \% \pm 10.1 \%$ (the reservoir strain), respectively. The number of cases requiring readjustment after automatic tracing were 14(9.6\%), 11(7.3\%), and 7(4.9\%) for LV, LA, and RA, respectively. For manual strain assessment, the success rate for LV, LA, RV, and RA were $149 / 152$ (98.0\%), 151/152(99.3\%), 138/152 (90.8\%), and 149/152 (98.0\%), respectively, and the GLSs were $-20.8 \% \pm 2.4 \%, 37.6 \% \pm 9.9 \%$ (reservoir strain), $-31.3 \% \pm 5.8 \%$, and $39.3 \% \pm 8.8 \%$ (the reservoir strain), respectively. 
Table 1

Clinical characteristics in all study patients

\begin{tabular}{|ll|}
\hline Variable & All study patients $(\mathbf{n}=\mathbf{1 5 2})$ \\
\hline Age, $\mathrm{y}$ & $40 \pm 11$ \\
\hline Male & $80(53)$ \\
\hline Height, $\mathrm{cm}$ & $165 \pm 8.2$ \\
\hline Weight, $\mathrm{kg}$ & $62.6 \pm 11.4$ \\
\hline Body surface area, $\mathrm{m}^{2}$ & $1.69 \pm 0.18$ \\
\hline Body mass index, kg/m ${ }^{2}$ & $22.8 \pm 2.7$ \\
\hline Heart rate, bpm & $71.2 \pm 10.8$ \\
\hline Systolic blood pressure, $\mathrm{mm} \mathrm{Hg}$ & $113 \pm 12$ \\
\hline Diastolic blood pressure, $\mathrm{mm} \mathrm{Hg}$ & $75 \pm 8$ \\
\hline LVEDV, ml & $110.2 \pm 24.7$ \\
\hline LVESV, ml & $41.4 \pm 10.8$ \\
\hline LVEF, \% & $62.0 \pm 7.5$ \\
\hline
\end{tabular}

\subsection{Comparison between automatic strain analysis and manual strain analysis}

\subsubsection{Comparison of LV global longitudinal strain}

For LV GLS, no significant difference was found in the results of automated strain analysis between two consecutive cardiac cycles $(-21.0 \% \pm 2.5 \%$ vs $-21.1 \% \pm 2.4 \%, \mathrm{~N}=145, \mathrm{P}=0.450)$, and they showed good correlation ( $r=0.854, P<0.001$, see Supplementary Table 1 and Fig. 3 ). Supplementary Table 2 and Fig. 4 showed that no difference was found in LV GLS values measured by two different methods $(-21.0 \% \pm 2.6 \%$ VS $-20.8 \% \pm 2.4 \%, N=144, P=0.230)$, and the correlation coefficients were $0.841(P<0.001)$.

\subsubsection{Comparison of RV free-wall longitudinal strain}

The RV free-wall (RVFW) longitudinal strain derived by the fully-automatic software had a significant difference compared to the manual measurement, which yielded a lower (less negative) strain $(-26.4 \%$ $\pm 4.9 \%$ vs. $-31.3 \% \pm 5.8 \%, N=137, P<0.001$, see Fig. 4). Poor correlation was found between RVFW strains obtained by two methods ( $r=0.246, P<0.005$, see Fig. 5). No significant difference was shown in the results of automatic analysis between different cardiac cycles $(-26.3 \% \pm 4.9 \%$ vs $-26.4 \% \pm 4.8 \% \mathrm{~N}=147$, $P=0.819)$, and they showed a good correlation ( $r=0.742, P<0.001$, see Fig. 3$)$. 


\subsubsection{Comparison of left atrial strain}

The left atrial function includes the reservoir, conduit, and booster pump function, which can be expressed by LASr (left atrial reservoir strain), LAScd (left atrial conduit strain), and LASct (left atrial contraction strain). The LASr and LAScd measured by the fully-automatic method had a statistically significant difference compared to the manual method, which yielded lower (less negative in LAScd) strains, while there is no significant difference in LASct. The correlations of LA strains obtained by two different methods were poor. The correlation coefficient ranged from a minimum of $0.242(P<0.001)$ for LASct, to a maximum of $0.545(P<0.001)$ for LAScd.

No significant difference was found in the results of automatic analysis between different cardiac cycles (LASr: $48.0 \% \pm 10.0 \%$ vs $47.7 \% \pm 10.2 \%, N=151, P=0.467$; LAscd: $-31.8 \% \pm 8.9 \%$ vs $-31.8 \% \pm 8.7 \%, N=151$, $P=0.920$; LASct: $-16.3 \% \pm 6.5 \%$ vs $-16.0 \% \pm 7.0 \%, N=151, P=0.506)$, and they showed good correlations between the two cardiac cycles (LASr $r=0.881, P<0.001$; LAScd $R=0.803, P<0.001$; LASct $R=0.751, P<$ $0.001)$.

\subsubsection{Comparison of right atrial strain}

Strains of RA were similar to those of LA, including RASr, RAScd, and RASct. The strains measured by the fully-automatic method had statistically significant differences compared to the manual method, which yielded higher RASr and RAScd (more negative) and lower RASct (less negative). Poor correlation was found between the two methods (see Fig. 5).

There was no significant difference in the results of automated strain analysis between two cardiac cycles (RASr: $41.1 \% \pm 10.1 \%$ vs $41.1 \% \pm 10.0 \%, P=0.936$; RAScd: $-28.1 \% \pm 9.4 \%$ vs $27.5 \% \pm 9.2 \%, P=0.387$; RASct: $-13.5 \% \pm 7.2 \%$ vs $13.0 \% \pm 6.0 \%, P=0.446 ; \mathrm{N}=146$ ), and they showed good correlations (RASr $r=0.785$, $P<0.001$; RAScd $r=0.595, P<0.001$; RASct $r=0.397$, $P<0.001$ ).

\subsection{Time of analysis}

The time for fully-automatic analysis of LV, LA, RV, and RA was significantly shorter $(17 \mathrm{~s}, 7 \mathrm{~s}, 9 \mathrm{~s}, 32 \mathrm{~s}$ per case, respectively) when compared with the manual method (91s, 51s, 49s, 54 s per case, respectively, $\mathrm{P}<$ 0.001, see Fig. 6).

\subsection{Reproducibility}

For intra-observer variations, the automatic method showed good agreement. The correlation coefficients of LV, LA, RV and RA were 0.999 (0.998-1.000), 0.999 (0.998-1.000), 0.988 (0.967-0.995), 0.997 (0.994$0.999)$, respectively, and the corresponding values of the manual editing method were 0.963 (0.909$0.985), 0.951(0.881-0.980), 0.954(0.886,0.982), 0.968(0.922-0.987)$, respectively.

The inter-observer variation for automatic analysis measured by ICC ranged from a minimum value of $0.968(0.922-0.987)$ for RA to a maximum value of 0.997 (0.991-0.999) for LV. For manual analysis, the 
inter-observer variation ranged from a minimum of 0.913 (0.793-0.966) for RV to a maximum of 0.938 (0.852-0.975) for LA (See Supplementary Table 3).

\section{Discussion}

The study's major findings can be summarized as follows: i) The results showed that the automatic analysis technique has high feasibility and good reproducibility. The success rate reis more than $94 \%$, and only less than $10 \%$ of the cases need to be readjusted. ii) Compared with the manual method, the automatic analysis is time-saving. The average time for LV was less than 20 seconds for automatic analysis, while the manual analysis takes more than 90 seconds. iii) The automatic analysis result of LV strain was consistent with the manual analysis. In contrast, the LA, RA, and RV results were significantly different between the two methods, and may not be used interchangeably.

\subsection{Ventricular strain analysis}

LV-GLS has been proved as a strong and independent predictor of cardiovascular events $[9,10]$. Various studies have proved that GLS is of more prognostic value than LVEF in chemotherapeutic[11], cardiac transplant[12], valvular disease patients[13]. The manual analysis of LV-GLS has been widely validated. In our study, the results of automatic strain analysis were consistent with those of traditional strain analysis but took a shorter time with higher repeatability.

For RVFW-GLS, the consistency between the two methods was poor. This may be due to the anatomical assumptions and default setting algorithms of the two software. The manual RVFW-GLS analysis was achieved using an LV-specific strain analysis package, while the automatic analysis embedded an RVspecific package. Anatomically, in contrast to the ellipsoidal shape of the LV, the RV appears triangular from the side and crescent in the cross-section. Mechanically, the shortening of the RV is greater longitudinally than radially. Twisting and rotation movement were less significant in RV than LV[14]. Therefore, the anatomical assumptions used in the RV-specific software were closer to the RV's real shape. Another reason for the vendor-to-vendor variability is the default settings of the software programs, such as the width of the myocardium to be tracked, the method of calculation of GLS (averaging of segments or length of line method)[15].

\subsection{Atrial strain analysis}

Similar to RVFW-GLS analysis, the atrial strains were achieved by using the LV-specific strain analysis package in manual analysis, while in the automatic analysis, the atrial strains were measured by using an LA-specific strain analysis package. The study showed a poor consistency in atrial strain measurement between the results of the two methods.

The reasons for the inconsistency may include the following aspects: i) Although the ROI width was set to $3 \mathrm{~mm}$ to fit the atria's thickness, the underlying myofiber architecture was different between atria and ventricles. The LA myocytes were irregularly arranged, and the alignment often changes at different 
depths with walls [16]. ii) The accuracy of LA strain measurement may also be affected by factors such as the thin-walled interatrial septum and the four pulmonary vein openings[17].

\subsection{Limitations}

The major limitation of the study is the lack of a gold standard for reference to compare the strain measurements. The study only compared the consistency between the two methods but lacked the comparison of their accuracy. Another limitation is that the study only included healthy patients. More investigations need to be done to determine the feasibility of the fully-automatic strain analysis in cardiac dysfunction patients, especially those with enlarged chamber or segmental wall motion abnormalities. The out-of-plane problem caused by the heart motion during the cardiac cycle exists in both methods, and the 3D-STE automatic analysis system would be a future trend.

\subsection{Conclusions}

Fully-automatic strain analysis is an efficient method with good feasibility and reproducibility. For LVGLS, the automatic analysis and manual analysis measurement results had no difference. For LA, RA, and RV, the GLS results of the two methods were inconsistent and cannot be used interchangeably. The discrepancies were caused by the different anatomical assumptions and default settings between the two methods.

\section{Declarations}

\section{Funding}

The authors declare that no funds, grants, or other support were received during the preparation of this manuscript.

\section{Competing Interests}

The authors have no relevant financial or non-financial interests to disclose.

\section{Author Contributions}

Guijuan Peng and Shuyu Luo performed the strain analysis and wrote the manuscript. Data acquisition was performed by Xiaofang Zhong and Xiaoxuan Lin. Yingqi Zheng performed the statistical analysis. Jinfeng Xu, Yingying Liu, and Lixin Chen conceived the idea for the manuscript and contributed to interpreting the data and revising the work. All the authors made the decision to submit. All authors read and approved the final manuscript.

\section{Ethics approval}


This study was performed in line with the principles of the Declaration of Helsinki. Approval was granted by the Ethics Committee of Shenzhen People's Hospital.

\section{Consent to participate}

Informed consent was obtained from all individual participants included in the study.

\section{Consent to publish}

The authors affirm that human research participants provided informed consent for the publication of their data.

\section{References}

1. Mollema SA, Delgado V, Bertini M et al. Viability assessment with global left ventricular longitudinal strain predicts recovery of left ventricular function after acute myocardial infarction. Circulation Cardiovascular Imaging 2010; 3: 15-23

2. Haeck ML, Scherptong RW, Marsan NA et al. Prognostic value of right ventricular longitudinal peak systolic strain in patients with pulmonary hypertension. Circ Cardiovasc Imaging 2012; 5: 628-636.

3. Medvedofsky D, Koifman E, Jarrett $\mathrm{H}$ et al. Association of Right Ventricular Longitudinal Strain with Mortality in Patients Undergoing Transcatheter Aortic Valve Replacement. Journal of the American Society of Echocardiography 2020; 33:

4. D 'Alto M, D 'Andrea A, Di SG et al. Right atrial function and prognosis in idiopathic pulmonary arterial hypertension. International Journal of Cardiology 2017; 248: 320

5. Erwan D, Albin B, Damien F. Value of left atrial strain: a highly promising field of investigation. European Heart Journal - Cardiovascular Imaging 2015; 356-357

6. Negishi K, Negishi T, Kurosawa K et al. Practical Guidance in Echocardiographic Assessment of Global Longitudinal Strain. JACC: Cardiovascular Imaging 2015; 8: 489-492

7. Lang RM, Badano LP, Mor-Avi V et al. Recommendations for Cardiac Chamber Quantification by Echocardiography in Adults: An Update from the American Society of Echocardiography and the European Association of Cardiovascular Imaging. Eur Heart J Cardiovasc Imaging 2015; 16: 233-270

8. Kawakami H, Wright L, Nolan M et al. Feasibility, Reproducibility, and Clinical Implications of the Novel Fully Automated Assessment for Global Longitudinal Strain. Journal of the American Society of Echocardiography 2020; 34:

9. Yang $\mathrm{H}$, Negishi $\mathrm{K}$, Wang $\mathrm{Y}$ et al. Echocardiographic screening for non-ischaemic stage $B$ heart failure in the community. European Journal of Heart Failure 2016; 18: 1331-1339

10. Florescu M, Magda LS, Enescu OA et al. Early Detection of Epirubicin-Induced Cardiotoxicity in Patients with Breast Cancer. Journal of the American Society of Echocardiography Official 
Publication of the American Society of Echocardiography 2014; 27: 83-92

11. Sera FK, TS, Farr, M, Russo, C; Jin, Z; Marboe, CC; Di Tullio, MR; Mancini, D; Homma, S. Left Ventricular Longitudinal Strain by Speckle-Tracking Echocardiography is Associated With TreatmentRequiring Cardiac Allograft Rejection. Journal of Cardiac Failure 2014; 20: 359-364

12. Pandis D, Sengupta PP, Castillo JG et al. Assessment of Longitudinal Myocardial Mechanics in Patients with Degenerative Mitral Valve Regurgitation Predicts Postoperative Worsening of Left Ventricular Systolic Function. Journal of the American Society of Echocardiography 2014; 27: 627638

13. Olsen NT, Sogaard P, Larsson H et al. Speckle-Tracking Echocardiography for Predicting Outcome in Chronic Aortic Regurgitation During Conservative Management and After Surgery. Jacc Cardiovasc Imaging 2011; 4: 223-230

14. Haddad F, Hunt SA, Rosenthal DN, Murphy DJ. Right Ventricular Function in Cardiovascular Disease, Part I: Anatomy, Physiology, Aging, and Functional Assessment of the Right Ventricle. Circulation 2008; 117: 1436-1448

15. Natalie AS, C, Lee JP, Msph CKM et al. A Comparison of Left- and Right-Sided Strain Software for the Assessment of Intraoperative Right Ventricular Function. Journal of Cardiothoracic and Vascular Anesthesia 2018; 32: 1507-1515

16. Schotten U, Verheule S, Kirchhof P, Goette A. Pathophysiological Mechanisms of Atrial Fibrillation: A Translational Appraisal. Physiological Reviews 2011; 91: 265-325

17. Marwick TH. Methods used for the assessment of LV systolic function: Common currency or tower of Babel? Heart (British Cardiac Society) 2013; 99:

\section{Figures}




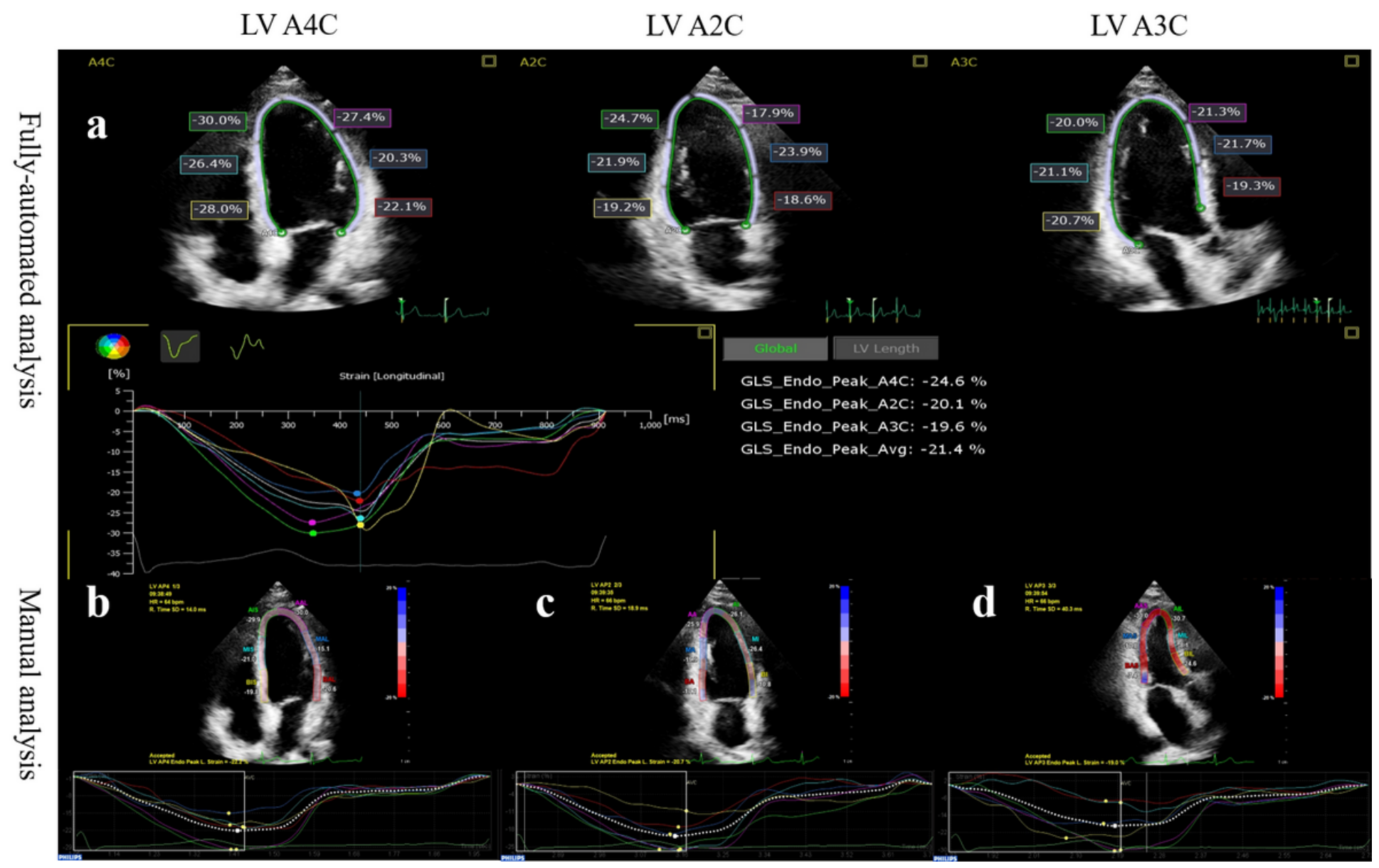

Figure 1

Left ventricular strain analysis results of the fully-automated analytical software and the manual analytical software. Upper row: For automatic analysis, videos of the three standard sections (the apical four-chamber, two-chamber, and three-chamber sections) were used for analysis. The software can automatically recognize, segment, and track the LV myocardium. The strain curves of 18 segments were generated, and the global LV-GLS and strains of each section were calculated simultaneously. Lower row: For manual analysis, after manually drawing the location of the apex, the anterior, posterior, lateral, and medial borders of the mitral annulus, the software tracked the LV myocardium frame by frame. The strain curves of each section and segment were presented. GLS, global longitudinal strain; LV A4C, left ventricular apical four-chamber section; LV A2C, left ventricular apical two-chamber section; LV A3C, left ventricular three-chamber section. 


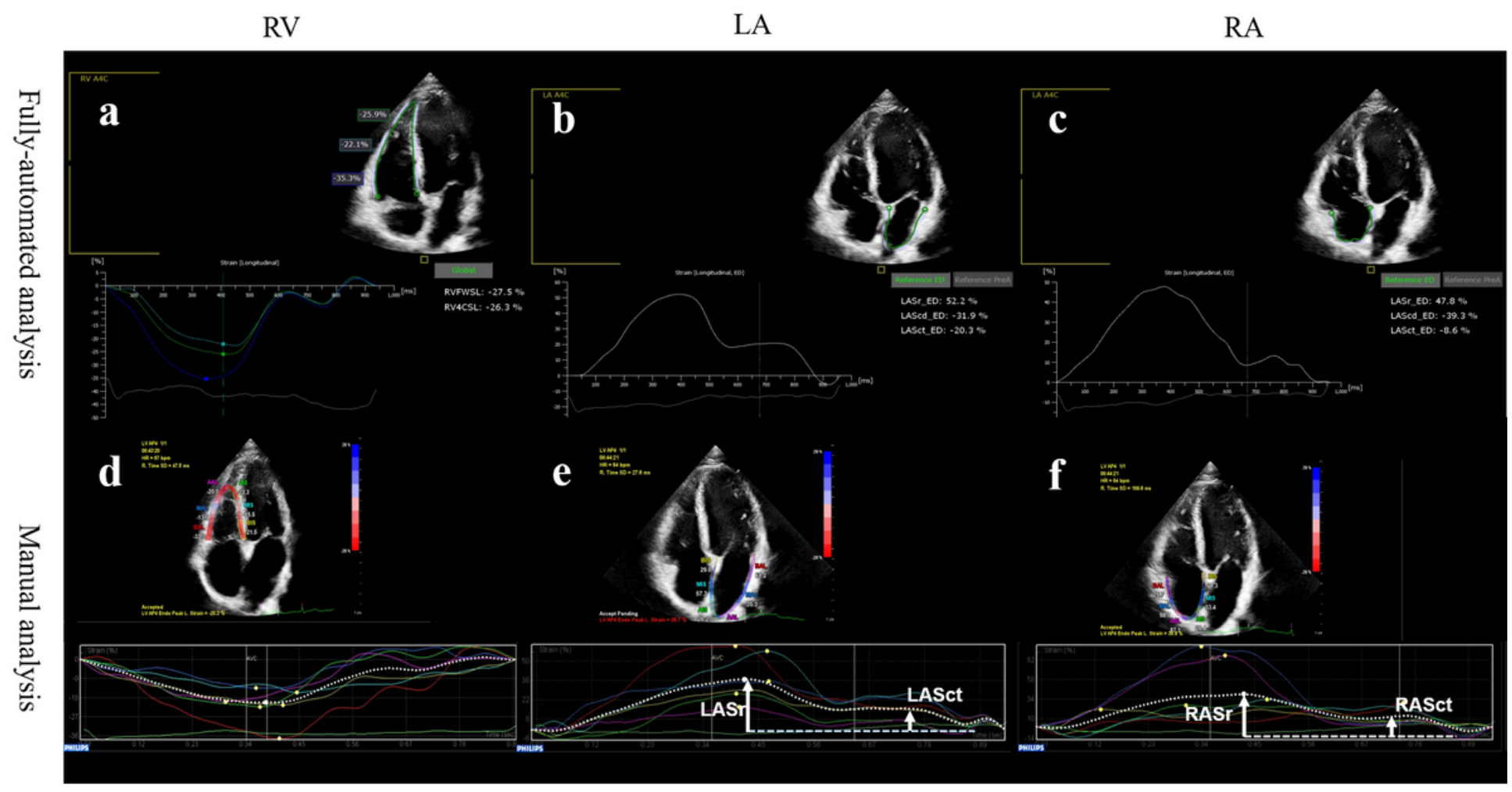

Figure 2

Right ventricular and atrial strain analysis results of the fully-automated analytical software and the manual analytical software. Upper row: For automatic analysis, the RV-specific and LA-specific analytic package was used for the RV (a) and LA (b) strain analysis, respectively. The RA strain was obtained using the LA-specific analytic package (c). Strains and strain curves were obtained. Lower row: For the manual method, all the analyses were achieved using the LV-based analytic package (d-f). The strain and strain curves of each segment were presented, and the right ventricular free-wall longitudinal strain was calculated by averaging the strains of three segments. RV, right ventricle; LASr, the left atrial reservoir strain; LASct, the left atrial contraction strain; RASr, the right atrial reservoir strain; RASct, the right atrial contraction strain. 

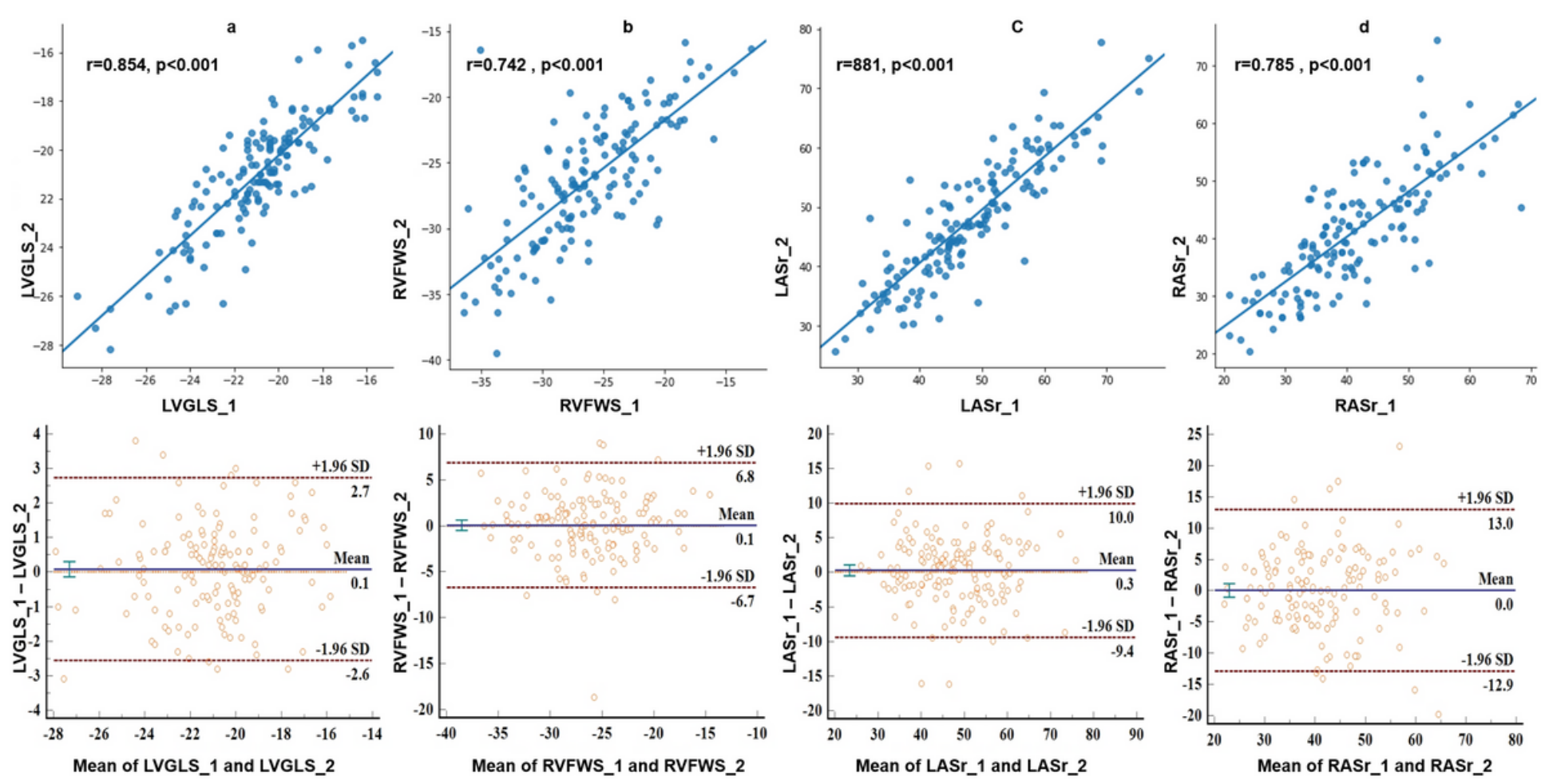

Figure 3

A linear correlation and Bland-Altman analysis of strain measurements between two consecutive cardiac cycles. (a) Left ventricular global longitudinal strain measurement; (b) Right ventricular free-wall longitudinal strain measurement; (c) Left atrial reservoir strain measurement; (d) Right atrial reservoir strain measurement.

a

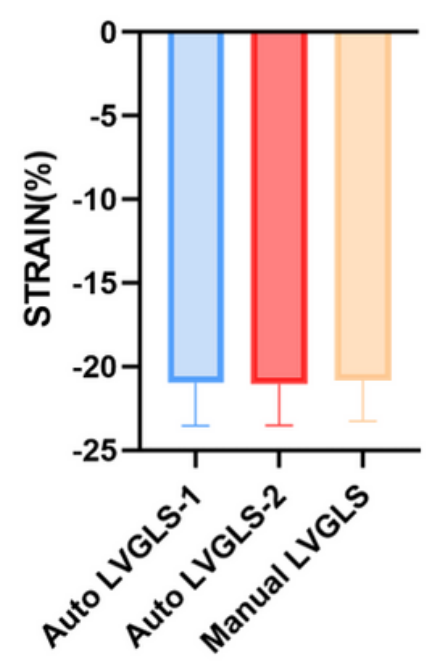

b

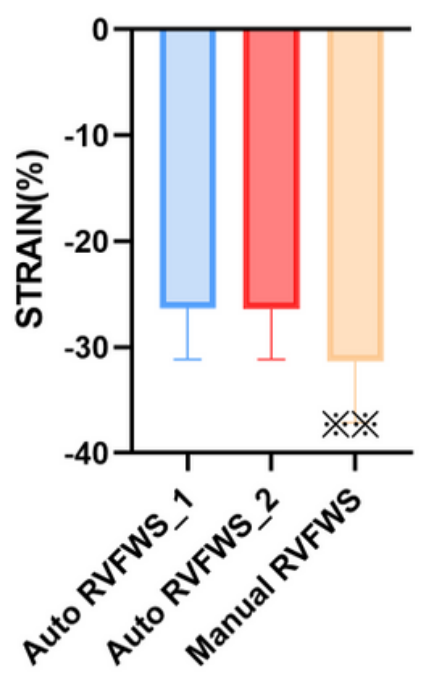

c

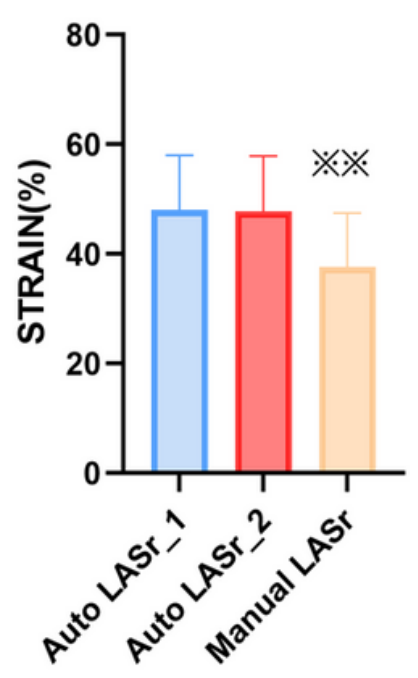

d

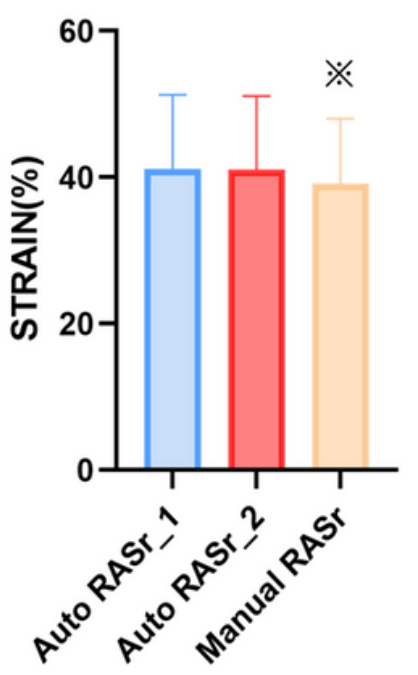

Figure 4 
Comparison of strains assessed by automatic (first cardiac cycle) vs automatic (second cardiac cycle) vs manual strain analytic software. No difference was shown in automatic analysis between different cardiac cycles. Between the two methods, there were no significant differences in LVGLS (a). Significant differences were noted in RVFWS (b), LASr (c), and RASr (d). Abbreviations: auto1, automatic analysis for the first cardiac cycle; auto2, automatic analysis for the second cardiac cycle; LVGLS, global longitudinal strain of left ventricle; RVFWS, right ventricular free-wall longitudinal strain; LASr, left atrial reservoir

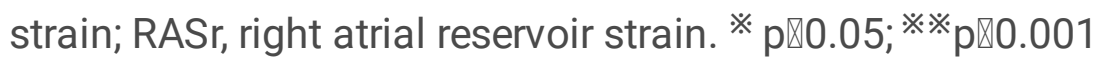
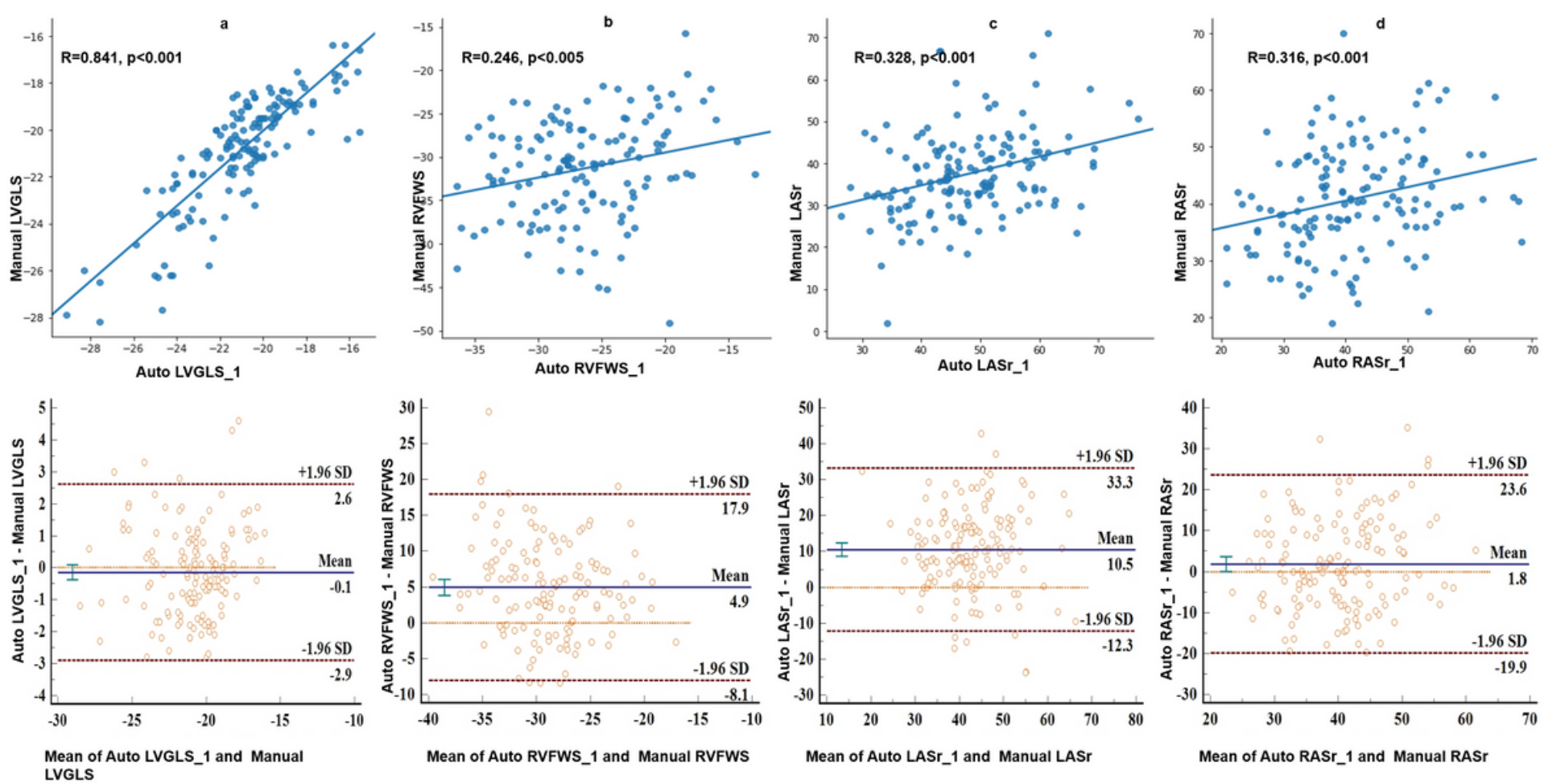

Figure 5

Linear correlation and Bland-Altman analysis of strain measurements between the automatic and manual method. (a) Left ventricular global longitudinal strain measurement; (b) Right ventricular free-wall longitudinal strain measurement; (c) Left atrial reservoir strain measurement; (d) Right atrial reservoir strain measurement. 


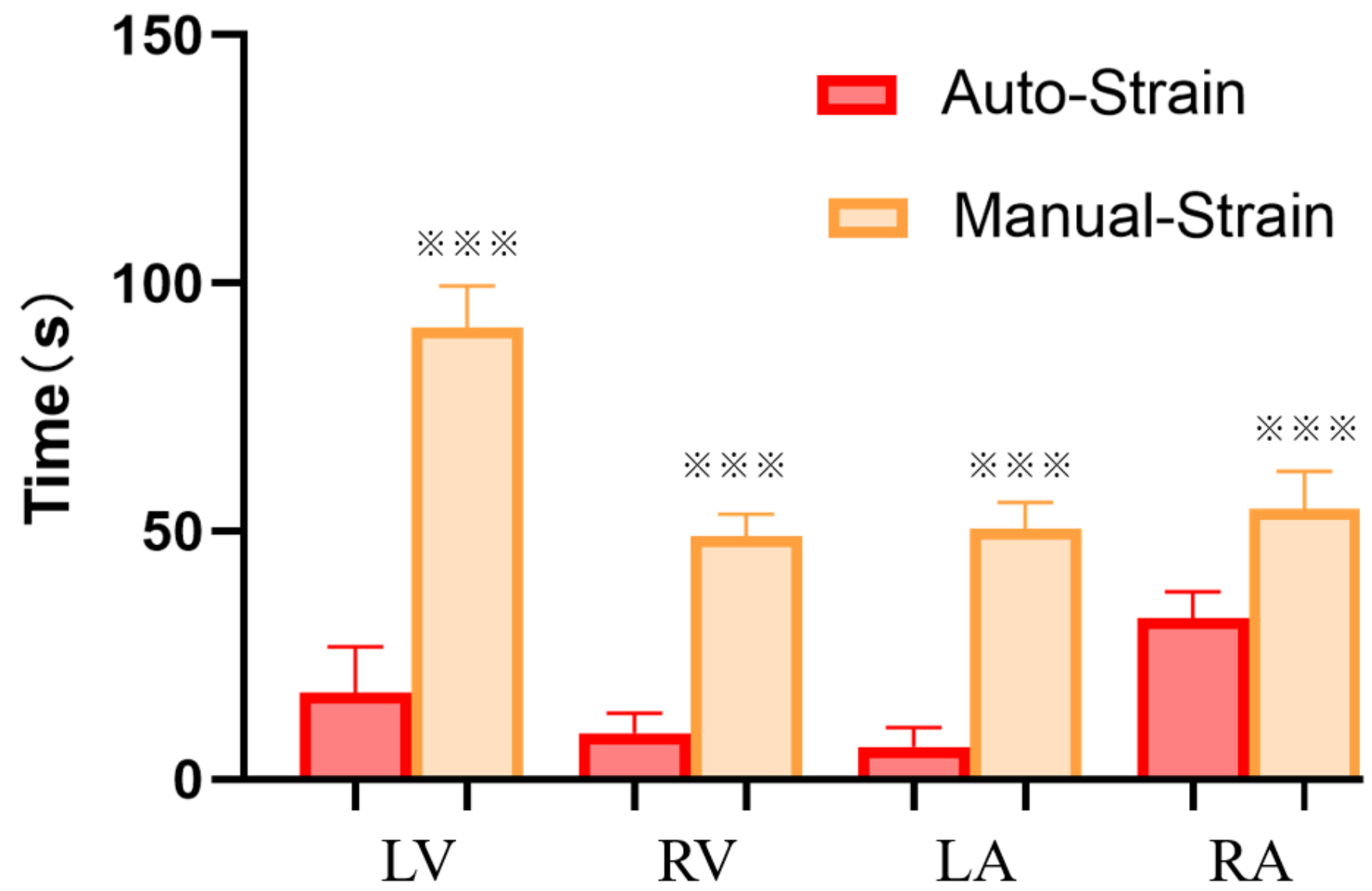

Figure 6

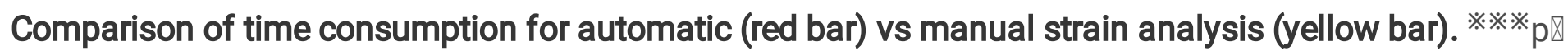
0.001

\section{Supplementary Files}

This is a list of supplementary files associated with this preprint. Click to download.

- SupplementaryMaterials.docx 